\title{
Unconventional energy: Seeking the ways to innovate energy science and technology
}

\author{
(C) Higher Education Press and Springer-Verlag GmbH Germany, part of Springer Nature 2018
}

With the energy and environmental problems becoming increasingly serious, pursuing ideal energy or its utilization ways has never been so urgent as it was. So far, tremendous efforts in the research of energy science and technologies have been conducted. However, there still exists a gap between researches and needs. This indicates that new energy or revolutionary technologies over existing ones should be fully explored. Meanwhile, some unconventional energy can be revisited and significantly improved from new view. Clearly, breakthrough in the area lies in the unconventional thoughts and game changing technical endeavors rather than gradual innovation.

In this thematic issue, 10 feature articles either from invited or selected contributions were assembled to collectively illustrate typical progresses in finding unconventional energy technologies. The topics cover a wide variety of newly emerging endeavors including electrochemical energy storage battery, lithium-ion battery, biofuel cell, solar thermoelectric generator, hydrogen generation, tidal energy driven reverse osmosis desalination, fusion energy, unconventional energy materials such as nanotube, thin film and liquid metal, etc. Lastly but not least, as also an important remedy and alternative aspect in innovating modern energy

Received May 2, 2018; online Jun. 2, 2018

Jing LIU (

Beijing Key Lab of Cryo-Biomedical Engineering and Key Lab of Cryogenics, Technical Institute of Physics and Chemistry, Chinese Academy of Sciences, Beijing 100190; School of Future Technology, University of Chinese Academy of Sciences, Beijing 100049; Department of Biomedical Engineering, School of Medicine, Tsinghua University, Beijing 100084, China

E-mail: jliubme@tsinghua.edu.cn

Zhixiong GUO ( $₫)$

Department of Mechanical and Aerospace Engineering, Rutgers, The State University of New Jersey, NJ 08854, USA

E-mail: guo@jove.rutgers.edu system, energy efficient sustainable human-building ecosystem was also particularly tackled.

Among the many advanced electrochemical energy storage technologies, redox flow batteries (RFB) is rather special owing to its outstanding merits in storing energy from intermittent renewable energy sources like wind and solar. Such system has favorable features over other battery technologies like solid state batteries, due to their safety and independent scaling of energy and power content. Critical issues that prevent such energy from large scale use lie in the low energydensity, low power density, and cost of components such as redox species and membranes. Stimming and his coworkers addressed such issues in their feature article by presenting inspiring concepts, new battery chemistries and cell configurations leading to cost effective RFB. They pointed out that promising answer lies in the cells chemistries that should be all-liquid, slurries or hybrids combining liquid, gas and solid phases together. Some favorable redox-systems, options of solvents as well as redox couples with entailing consequences were elucidated. This work provides a directional guidance to search for further ideal RFB which simultaneously owns the properties of high energy- and power-density, long-time stability and low capital costs.

Batteries have many different forms indeed. Lithiumion battery is such an attractive compact option. Direct integration of lithium-ion battery (LIB) with electronic devices on the same $\mathrm{Si}$ substrate is a core to significantly miniaturize autonomous micro systems. Huang and his coworkers investigated the feasibility of utilizing thermal $\mathrm{SiO}_{2}$ film as the barrier layer through electrochemical characterization and X-ray photoelectron spectroscopy. They clarified the electrochemical performances of thermal $\mathrm{SiO}_{2}$ film and demonstrated that $30-\mathrm{nm}$ film is effective to act as the barrier layer for direct integration of LIB with the electronic devices. This work illustrates the emerging technique to improve 
battery on chip via microfabrication way and would advance the micro system integrated with an electronic device.

With environmentally friendly feature, biofuel cell belongs to an important energy category which has received increasing attentions over the past few years. Through introducing the latest star material graphene, $\mathrm{Xu}$ et al. proposed to enhance the performance of a membraneless enzymatic biofuel cell through developing a nitrogen-doped graphene approach. Some new findings were made such as that the electron accepting nitrogen sites in the graphene structure would enhance the electron transfer efficiency between the mediator (redox polymer), redox active site of the enzymes, and electrode surface. They also observed the best performance in terms of power output and current density of biofuel cell when the bioanode was modified with highly doped N-graphene. This work would provide fundamental as well as practical guidance for future work.

With straightforward style in directly translating the thermal energy into electricity based on the Seebeck effect, solar thermoelectric generator offers an unconventional way for convenient utilization of green energy from the concentrated sunlight. Nevertheless, many fundamental or practical issues are not clear enough to further improve this technology. Li and Xuan's team clarified the effect of non-uniform illumination on the performance of solar thermoelectric generators through a three-dimensional finite element modeling and simulation. And some interesting suggestions such as reducing the number of the thermoelectric legs for nonuniform solar illumination were given to significantly increase the performance of the thermoelectric generator.

Except for direct use of solar energy, hydrogen also offers an ideal green energy and tremendous efforts were made to find better ways for its renewable production and storage. In this regard, nanotechnology enhanced photoelectrochemical (PEC) water splitting is rather promising. Aiming for an improved hydrogen fuel production, Ali et al. made material endeavor for innovative photoelectrodes through synthesizing the vertically aligned $\mathrm{TiO}_{2}$ nanotube arrays decorated with $\mathrm{WO}_{3}$ particles. A group of characterization techniques found that, such modified nanotubes facilitated a more efficient utilization of solar light for water splitting. And the optimal photoelectrode evidently improved the photo conversion efficiency and hydrogen generation compared to that of the pure nanotubes without decoration. This study will be of interest of materials scientists and researchers in environment and energy technology field.

In recent years, the room temperature liquid metal (RTLM) is quickly emerging as an important functional material in a variety of energy areas owing to its many outstanding performances. Aiming to mold next generation unconventional thermal delivery or management technologies and further advance the liquid metal cooling, Yang and Liu presented a perspective article which outlined a new conceptual scientific category termed as combinatorial liquid metal heat transfer science. The work and the fundamental principles proposed therein illustrated that through combining the advantages between liquid metal and existing heat transfer technologies, a group of innovative thermal systems can be enabled which would help break up the bottleneck limit facing a single conventional system.

Apart from the pure liquid metal, a recent understanding in the area is that the hydrodynamic characteristics of hybrid fluid made of liquid metal/aqueous solution are very unlike those single fluids. Such knowledges are elementary in the design and operation of conductive flow as well as for better understanding of the interfacial phenomena in a variety of newly emerging areas such as chip cooling, energy harvesting, soft robot, and biomedical practices, etc. For better advancing the area, Liu and his coworkers presented a comprehensive review on the unconventional hydrodynamics involved in modeling and interpreting the liquid metal/aqueous solutions from experimental, theoretical and simulation aspects. Typical experimental phenomena and basic working mechanisms regarding the complex liquid metal flow are illustrated, followed by the movement and deformation theories to explain these phenomena. Several representative simulation methods are proposed to tackle the governing function of the electrohydrodynamics. The prospects and challenges as raised would offer an insight into the new physics of the hybrid fluids under applied fields.

As we know, the core goal of developing various energies is always for human life. Zhang et al. presented such an example by proposing to use tidal energy to realize a reverse osmosis $(\mathrm{RO})$ desalination system to tackle water crisis. Economic evaluation indicated that, such tidal energy RO system can save evident water cost in comparison with the conventional RO system. Utilizing the mechanical energy produced by the tidal energy through the hydraulic turbine suggests a feasible way to directly drive the $\mathrm{RO}$ unit. Clearly, this work also reminds further efforts to find more ways to fully use energy in nature.

The above contents give some latest examples towards finding new and green energy. In fact, unconventional energy refers to any game changing energy. Fusion energy is just a very unusual example and has been considered as the ultimate energy source, which does not contribute to climate change compared with conventional fossil fuel. Huang and Li presented a review on the main developments of magnetic confinement fusion with emphasis on confinement systems as well as challenges of materials related to superconducting magnet and plasma facing components. Their work would help better understand the fusion roadmap 
towards commercial fusion power plant even ultimate energy source.

As is not always recognized, energy efficient sustainable human-building ecosystem (SHBE) has in fact always been an important issue in modern energy system. Tao and his coworkers contributed an inclusive review on latest studies along this direction. Unlike existing ideas that just focus on developing new energy saving technologies, they emphasized that synergistic podium should be the way to subdue barriers in engineering, architectural design, social and economic perspectives that hinder wider application, adoption and subsequent performance of sustainable building solutions by recognizing the essential role of human behaviors within building-scale ecosystems. Collaborative efforts from various disciplines such as engineering, software design, sociology, and law should be made for transformative technologies, designs and methods of adoption for future design, construction and operation of sustainable buildings. This work offers inspiring thoughts for developing future energy system.

We hope you will enjoy reading the concepts, proposals, or specific strategies contained in this special collection of review and research articles on the topics of unconventional energy. Facing ever tougher challenges in this era of energy issues on earth, struggling to find breakthrough technologies in energy utilization and generation is the way to solve the problem. More unconventional technologies are urgently waiting to be addressed.
Editor's bio-sketches:

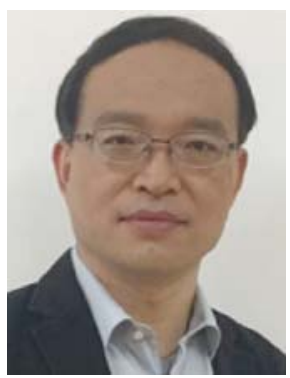

Dr. Jing Liu is a jointly appointed Professor of Tsinghua University (THU) and Technical Institute of Physics and Chemistry, Chinese Academy of Sciences. He received his double B.S. degrees in both Engineering and Physics, and Ph.D. in thermal science with speciality on bioengineering, all from THU. Dr. Liu had ever performed visiting researches at Purdue University and Massachusetts Institute of Technology. He works at the interdisciplinary areas among liquid metals, biomedical engineering, and thermal science and is an author of nine popular books with one reprinted five times, and about 460 journal articles with over 20 selected as journal cover or back cover image story. Dr. Liu pioneered a group of very fundamental discoveries on liquid metal matters which were frequently featured over the world. His researches on liquid metal chip cooling, printed electronics, biomaterials, and soft machines initiated many important technologies. He is a recipient of numerous awards like: The William Begell Medal, R\&D 100 Award Finalist, Best Paper of the Year Award from ASME Journal of Electronic Packaging, CCTV 2015 Top Ten Figures in Science and Technological Innovation, and five times highest teaching award from CAS, etc. E-mail: jliubme@tsinghua. edu.cn.

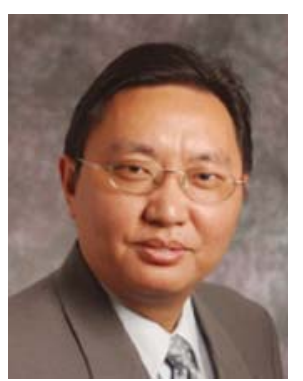

Dr. Zhixiong Guo is a Professor of Mechanical and Aerospace Engineering at Rutgers University-New Brunswick, USA. $\mathrm{He}$ is a Fellow of ASME. His research spans a wide area in heat and mass transfer and energy system, with notable expertise in thermal radiation, laser manufacturing, micro/nanoscale phenomena, and optical sensing. He has published over 200 research articles in archival journals and conference proceedings. $\mathrm{He}$ is the managing editor for Heat Transfer Research and an associate editor for Journal of Heat Transfer (2013-2019). He was bestowed the Board of Trustees Award for Excellence in Research in 2018, the university's highest honor for outstanding research contributions to a discipline or to society. E-mail: guo@jove. rutgers.edu. 